\title{
Multiparameter Approach for Damage Propagation Analysis in Fiber Reinforced Polymer Composites ${ }^{\dagger}$
}

\author{
Claudia Barile, Caterina Casavola, Giovanni Pappalettera* and Paramsamy Kannan \\ Vimalathithan \\ Dipartimento di Meccanica, Matematica Management, Politecnico di Bari, Via Orabona 4, 70125 Bari, Italy; \\ claudia.barile@poliba.it (C.B.); casavola@poliba.it (C.C.); pk.vimalathithan@poliba.it (P.K.V.) \\ * Correspondence: giovanni.pappalettera@poliba.it \\ + Presented at 1st International Electronic Conference on Applied Sciences, 10-30 November 2020; Available \\ online: https://asec2020.sciforum.net/.
}

Published: 10 November 2020

\begin{abstract}
Assessing damage evolution in Carbon Fiber Reinforced Polymer (CFRP) composites is an intricate task due to the complex mechanical response of the composites. The acoustic emission technique $(\mathrm{AE})$ is a non-destructive technique based on the recording of sound waves generated inside the material as a consequence of the presence of active defects. Proper analysis of the recorded waves can be used for monitoring damage evolution in many materials including composites. The acoustic track associated with the entire loading history of the sample or the structures is usually followed by using some descriptors such as the amplitude of the sound waves, the number of counts and so on. In this paper the acoustic emission in CFRP single lap shear joints will be monitored by using a multiparameter approach based on the contemporary analysis of multiple features such as ASL, Initiation Frequency, Reverberation Frequency and so on to understand if a proper combination of them can be adopted for a more robust description of damage propagation in CFRP structures. For selecting the best features, Principal Component Analysis (PCA) is used. The selected features are classified into different clusters using Fuzzy c-means (FCM) data clustering for analyzing the damage modes.
\end{abstract}

Keywords: acoustic emission; CFRP; fuzzy c-means; principal Component Analysis (PCA); initiation frequency; reverberation frequency; Single Lap Shear (SLS);

\section{Introduction}

Acoustic Emission (AE) technique is based on the detection and interpretation of sound waves, caused by rapid internal displacements in a material, travelling at an ultrasonic velocity [1,2]. The formation and propagation of cracks due to different damage sources are the sources of these acoustic signals. In simple words, when a material is locally deformed irreversibly, it releases energy in the form of elastic waves and the $\mathrm{AE}$ technique is based on the analysis of these elastic waves. The characteristics of these elastic waves are studied in their frequency domain or time-frequency domain using different types of waveform analysis [3].

The waveform analysis involves post-processing of the acoustic signals after acquisition. This post-processing sometimes requires high computational power and consumes lots of storage space. Especially, when the continuous acoustic signals are studied in their time-frequency domain, the data processing consumes lots of time. For these reasons, the acoustic signals can be characterized in terms of their energy, peak amplitude/frequency, duration, risetime and so many other different parameters. These parameters are derived from the acoustic signals that can define the characteristics of the acoustic source. 
There exists a longstanding debate on which are the most relevant parameters that can be used for describing the damage characteristics most efficiently [3]. Peak amplitude and peak frequency of the waveform often standout as the best parameter for defining the characteristics of the acoustic source. In the last 5 years, several researchers have evidently proved that the peak amplitude and peak frequency of the acoustic waveform mislead information about the damage characteristics, especially in Fiber Reinforced Polymer (FRP) composites [4-6]. This is because several damage characteristics release acoustic waveforms with the same peak frequency. For instance, in a Carbon Fiber Reinforced Polymer (CFRP) material, the fiber breakage during a static tension loading releases acoustic signals with the same frequency as the interlaminar crack propagation [4].

Analyzing the acoustic emission signals only based on their peak frequency is the reason for this anomaly. To avoid this erroneous description of the damage characteristics of the acoustic source, a multiparameter approach is required. The debate resumes once again on the conception of the multiparameter approach: what are the parameters that can be selected? Few researchers have used risetime and rise angle (RA) of the acoustic waveforms [7], while some others used absolute energy [8]. A definite set of parameters has not been defined specifically as the optimal set of parameters for analyzing the damage characteristics.

Recently, Guel et al. [9] used a multiparameter approach by integrating the acoustic results recorded from two different types of piezoelectric sensors. The parameters they used for this analysis are centroid frequency, peak frequency and energy. Although these parameters have been commonly used by several researchers in the past, Guel et al. used an exploratory data analysis for selecting these parameters [9]. Nonetheless, the parameters they have used for analysis are quite common in literature.

In this research work, the Principal Component Analysis (PCA) is used for identifying the sets of parameters for analysis. The initial parameter set which is considered are the peak amplitude, Absolute Signal Level (ASL), Initiation Frequency (I-Frequency), Peak Frequency (P-Frequency), Average Frequency (A-Frequency) and Reverberation Frequency (R-Frequency). These parameters may represent the characteristic features of the waveform. Besides, during the preliminary analysis and our previous research works [6], the authors have identified that the acoustic signals observed from different damage modes are highly unsymmetrical. The propagation of the acoustic signals released from the different damage sources may be responsible for the asymmetry of these signals. For this reason, the I-Frequency and R-Frequency alongside with other AE signals are considered for analysis.

The selected signals are clustered using the Fuzzy c-means data clustering technique into different classes. In this research work, the acoustic signals used are recorded from CFRP laminates bonded in Single Lap Shear (SLS) configuration, which are subjected to a static tensile load. An attempt has been made to correlate the AE parameters, which are selected using PCA and clustered using Fuzzy c-means, with the different damage modes. The novelty of this work is the usage of IFrequency and R-Frequency, which poorly investigated in literature for damage characterization in CFRP.

The introduction about CFRP composites and their damage modes can be found in several standard textbooks and literature [10-12]. Similarly, the basic introduction about the AE technique and its application on the damage propagation analysis in CFRP can also be found in standard textbooks and several review articles [2,3,13-15]. For avoiding any redundant information, basic introductions are not provided in this section. The definitions of the AE parameters which are used in this study are presented in the subsequent sections.

\section{Experimental Procedure}

\subsection{Materials}

CFRP specimens in Single Lap Shear (SLS) configuration are used in this study. The CFRP prepreg laminates are prepared by impregnating high strength carbon fibers in the epoxy matrix (SATTI CIT CC206 ER 460). The carbon fibers are woven in the epoxy matrix in a stitched 
configuration. The laminate plies which have a nominal thickness of $0.244 \mathrm{~mm}$ are cured by the autoclave method. The upper and lower adherend for the SLS configuration is prepared by the autoclave method. Then a high-strength epoxy adhesive EA9395 with a shear strength of $25 \mathrm{MPa}$ and a peel strength of $65 \mathrm{MPa}$ is used for bonding the upper and lower adherend in the SLS configuration. The dimensions of the specimens, the laminate layup configuration and other dimensional details are presented in Table 1. A total of 3 specimens are tested for this research work and are named SLS 1, SLS 2 and SLS 3.

Table 1. SLS Specimen Nomenclature, Geometry and Configurations.

\begin{tabular}{ccccc}
\hline \multicolumn{5}{c}{ Upper Adherend } \\
\hline Length $l_{u}(\mathrm{~mm})$ & Width $b_{u}(\mathrm{~mm})$ & Thickness $h_{u}(\mathrm{~mm})$ & No. of Plies & Stacking Sequence \\
\hline $101.6 \pm 0.11$ & $25.33 \pm 0.12$ & $1.3 \pm 0.05$ & 5 & $+45 /+45 /+45 /-45 /+45$ \\
\hline \multicolumn{5}{c}{ Lower Adherend } \\
\hline Length $l_{l}(\mathrm{~mm})$ & Width $b_{l}(\mathrm{~mm})$ & Thickness $h_{l}(\mathrm{~mm})$ & No. of Plies & Stacking Sequence \\
\hline $101.6 \pm 0.09$ & $25.33 \pm 0.14$ & $6.4 \pm 0.12$ & 26 & $+45 /[+45 /-45]_{12} /+45$ \\
\hline \multicolumn{5}{c}{ Overlapping Region } \\
\hline Length $l_{\text {or }}(\mathrm{mm})$ & Width $b_{\text {or }}(\mathrm{mm})$ & Thickness $h_{\text {or }}(\mathrm{mm})$ \\
\hline $26 \pm 0.12$ & $25.33 \pm 0.25$ & $8.5 \pm 0.11$ \\
\hline
\end{tabular}

\subsection{Experimental Setup}

Two narrowband general-purpose piezoelectric sensors R-30 $\alpha$ (Physical Acoustics, MISTRAS Group, Princeton Junction, NJ, USA) are mounted on the SLS specimens for recorded the acoustic signals. The sensors have an operating range of $150 \mathrm{kHz}$ to $400 \mathrm{kHz}$. The sensors are mounted on the specimens at a distance of $40 \mathrm{~mm}$ from the center. The acoustic events generated between this distance, which covers the adhesive overlap region, is recorded for this study. A thin layer of silicone gel separates the piezoelectric transducer surface and the specimen for improving the acoustic coupling and also to avoid recorded any reverberating signals. All the AE signals are amplified by $40 \mathrm{~dB}$ using a 2/4/6 AE pre-amplifier. The acoustic waveforms are recorded at a sampling rate of 1 MSps (1 mega sample per second). The threshold for acquisition is set as $40 \mathrm{~dB}$.

For the static tensile loading, the ASTM D5868-Standard Test Method for Lap Shear Adhesion for Fiber Reinforced Plastic (FRP) Bonding is followed [16]. The static tensile loading is applied in a displacement-controlled mode at a crosshead displacement speed of $13 \mathrm{~mm} / \mathrm{min}$. The SLS specimens carry most of the load in the adhesive overlap region under the displacement-controlled testing mode. All the tests are carried out in the INSTRON Servo-Hydraulic testing machine, which has a loading capacity of $100 \mathrm{kN}$.

\subsection{Acoustic Emission Features}

The acoustic emission descriptors considered for this study are explained in this section. First, the most commonly used parameter Peak Amplitude is used. It represents the largest voltage peak of the recorded AE signal $\left(U_{\max }\right)$ with respect to the reference voltage $\left(U_{r e f}\right)$ set at the pre-amplifier. It is measured in decibels $(d B)$ and it can be expressed as,

$$
A=20 \log \frac{U_{\max }}{U_{\text {ref }}}
$$

Unlike the peak amplitude, which only represents the largest voltage peak, the average signal level (ASL) measures both the negative and positive amplitudes in an acoustic signal with equal weight. In simple words, it provides the average level of the signal in decibels. It can be expressed in volts as,

$$
A S L_{v}=\frac{1}{T} \int_{t_{0}}^{t_{0}+T}\left|V_{i}\right| d t=\frac{1}{N} \sum_{n=1}^{N}\left|V_{i}(n)\right|,
$$

where, $N$ is the total number of discretized signal points, $V_{i}$ is the transient voltage and $T$ is the duration of the signal. The ASL in $\mathrm{dB}$ can be expressed as, 


$$
A S L=20 \log \left(\frac{A S L_{v}}{1 \mu V}\right),
$$

Initiation Frequency (I-Frequency) measures the characteristic frequency of the acoustic signal before the peak amplitude is recorded. It is the ratio of the total number of acoustic counts before the peak amplitude (P-Counts) and the duration of the signal for that same instant (Risetime).

$$
\mathrm{I}-\text { Frequency }=\frac{\text { P. Counts }}{\text { Risetime }},
$$

Reverberation Frequency (R-Frequency) is the exact opposite measure of the I-Frequency. It measures the characteristic frequency of the signal after the peak amplitude. It is the ratio of the number of counts after the peak amplitude (Ringdown Counts) and the duration of the signal after that same instance.

$$
\mathrm{R}-\text { Frequency }=\frac{\text { Ringdown Counts }}{\text { Duration }- \text { Risetime }},
$$

Average Frequency (A-Frequency) is the ratio of the total number of counts recorded above the threshold of acquisition and the duration of the signal.

$$
A-\text { Frequency }=\frac{\text { Counts }}{\text { Duration }},
$$

Peak frequency of the AE signal is the frequency with the largest magnitude when the signal coefficients are analyzed in their frequency domain using Fast Fourier Transform (FFT).

These are the parameters that are considered for this study. The method of selecting the best parameters among the aforementioned parameters and the procedure followed for clustering the selected parameters are presented in the next section.

\subsection{Methodology for Data Clustering}

The different $\mathrm{AE}$ features are the characteristic representation of the acoustic waveform. Many of these $\mathrm{AE}$ features are closely in relation to one another. For example, the absolute energy of the $\mathrm{AE}$ signal and the ASL are directly in relationship with the RMS voltage. Absolute energy is directly proportional to the square of the RMS voltage, while ASL is in analogous with RMS voltage with the only difference being RMS voltage is measured in $m V$ or $\mu V$, whereas ASL is measured in $\mathrm{dB}$. In that context, using both RMS voltage and ASL for analyzing the signal characteristics is redundant.

The Principal Component Analysis (PCA) is the multivariable data reduction technique. The core idea of the PCA is to reduce the dimensionally of the data set, which has a large number of interrelated variables, which is essentially the case in this research work. The data reduction is done while retaining as much as possible of the variation present in the data set. The data reduction is achieved by reducing the data dimensions into new correlated features, called principal components, which are minimally correlated.

These principal components form a symmetric matrix, with the eigenvectors of the matrix forms the elements of the matrix. These eigenvectors can be defined as the characteristic vectors of the matrix. They are unique in a way that they remain directionally invariant under linear transformation by its parent matrix. The other details about this analysis can be found in the source article presented by Hotelling [17]. The procedure for calculating the principal components is summarized by Maćkiewicz and Ratajczak [18].

The multi-parameters are reduced using PCA and the characteristic parameters are selected for analysis. Then these selected parameters are clustered using the Fuzzy c-means (FCM) data clustering method. The FCM is a data clustering method for any 2-dimensional data. The dataset is clustered into a predefined number of clusters in FCM [19]. Unlike other data clustering methods, in which all the datapoints belong to only one cluster, in FCM, the datapoints belong to all the clusters to some degree. The degree of membership is based on the distance between the datapoint and the centroid of each cluster. The datapoint has a large degree of membership with the cluster with the closest centroid, while it has a smaller degree of membership with the cluster with the farthest centroid. 
The assignment of centroids and the datapoints to each cluster is determined by the objective function. By iterating, the distance from any given data point to a cluster center weighted by the membership of that data point in the cluster.

The detailed procedure for performing FCM on a dataset can be found elsewhere. Both the PCA and FCM are popular data analysis methods and their procedures are described in several literature over the years [17-19]. Thus, they are not provided here. Both FCM and PCA are carried out in MATLAB $^{\oplus}$; FCM is supported by the Fuzzy logic toolbox in MATLAB ${ }^{\circledR}$.

\section{Results and Discussions}

The AE data from testing SLS specimens SLS 1, SLS 2 and SLS 3 are recorded using the pair of piezoelectric sensors. The parameters mentioned in Section 2.3 are derived from the PAC PCI-2 data acquisition system.

\subsection{PCA for Data Reduction and Selection of AE Features}

The input parameters peak amplitude, ASL, I-Frequency, R-Frequency, P-Frequency and AFrequency are reduced into their eigenvector matrix using the PCA. The variances of the eigenvalues of the eigenvector matrix or the different principal components are also calculated. The scree plots describing the variance of different principal components are presented in Figure 1.

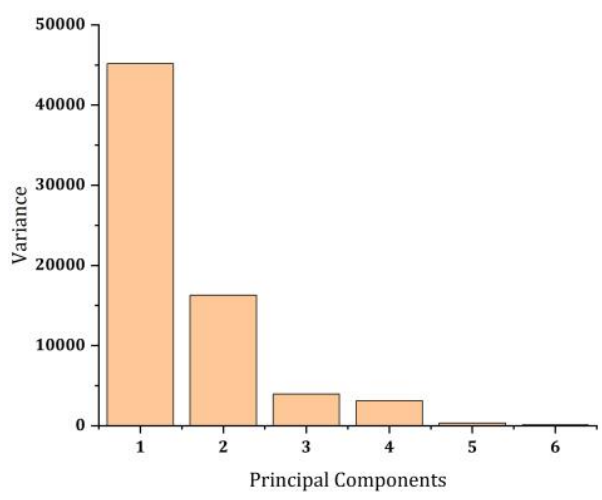

(a)

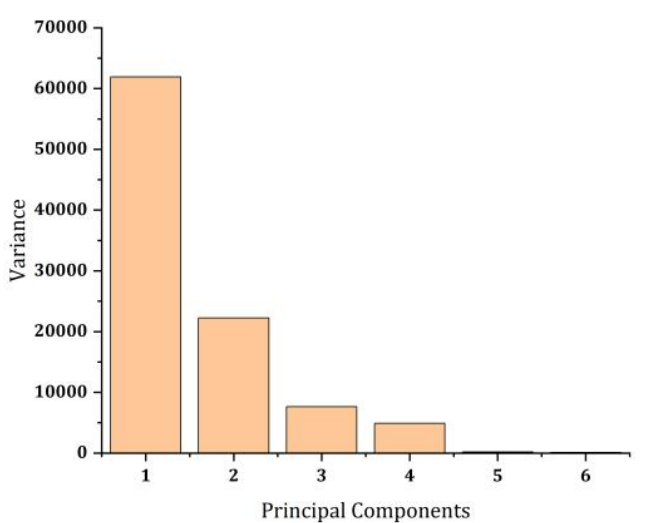

(b)

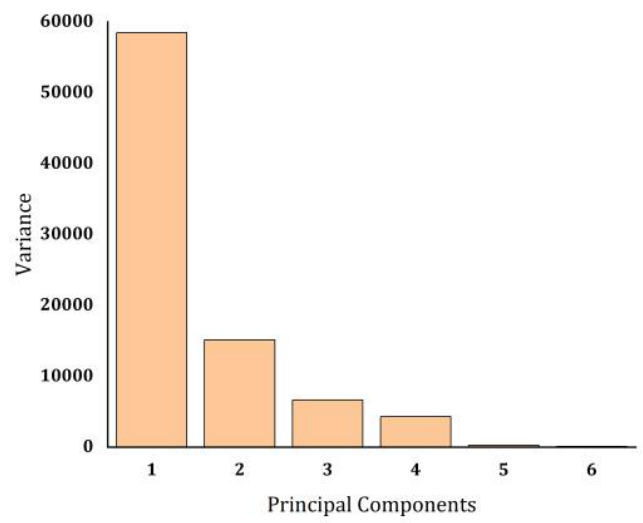

(c)

Figure 1. Scree Plot for different Principal Components for the dataset of (a) SLS 1 (b) SLS 2 and (c) SLS 3. 
The variances of the first and second principal components are significantly larger than the remaining components combined together. Thus, dataset is reduced only into the first two principal components PC 1 and PC 2.

For the reduced data, the eigenvalues of the principal components PC 1 and PC 2 of all the AE parameters are presented in Figure 2. It can be observed that the peak amplitude, peak frequency and amplitude form a group with the eigenvalues of PC 1 and PC 2 close to each other. Therefore, a representative AE parameter from this group is selected for further analysis. In this work, the peak amplitude is selected as the representative from the group.

It can also be observed from Figure 2 that the I-Frequency, R-Frequency and A-Frequency have large variations in their eigenvalues for PC 1 and PC 2 and cannot be constituted into a group. This means that there exists a large variation in these parameters. Therefore, the I-Frequency and RFrequency are considered for the analysis.

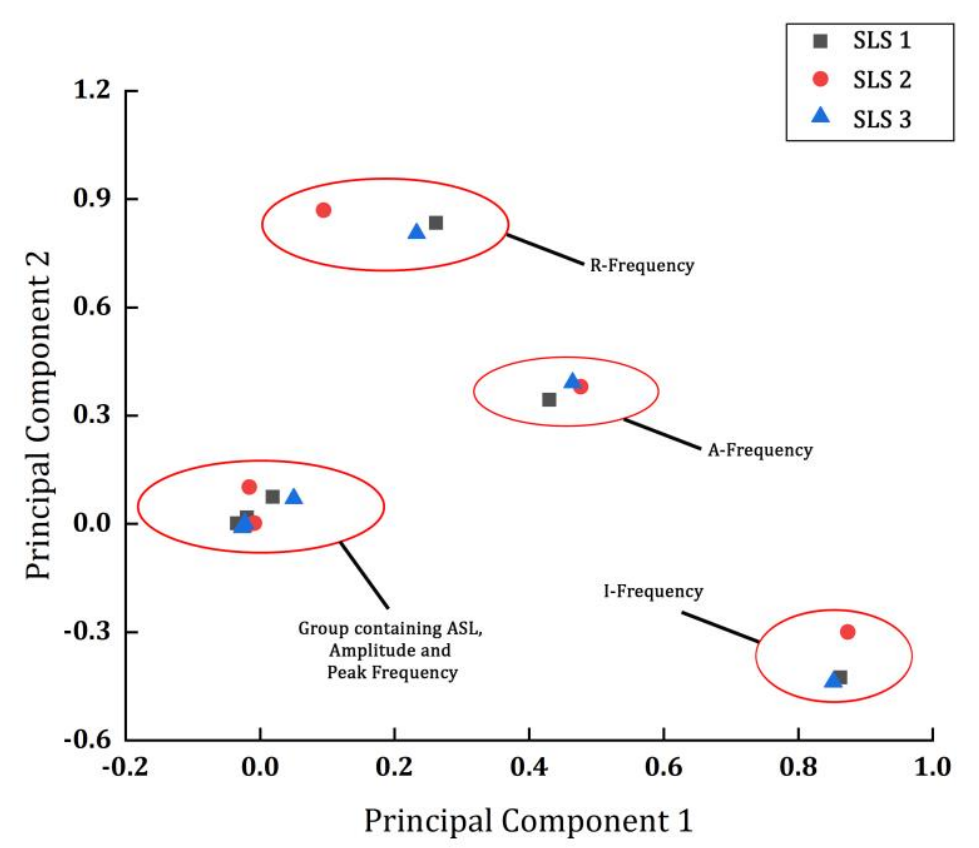

Figure 2. Principal Components PC 1 and PC 2 for all the parameters selected for all specimens.

From the PCA results, three parameters from the initially assigned group of six parameters are selected: Amplitude, I-Frequency and R-Frequency. Instead of going through a rigorous process of analyzing all the parameters and finding the optimal parameters for analysis, PCA reduces the dimensionality of the dataset into three parameters. For comparison purposes, the amplitude from the group of ASL, Amplitude and P-Frequency is compared from I-Frequency and R-Frequency.

There is a specific reason for comparing the Amplitude with these two parameters. For several years in the research of damage assessment using $\mathrm{AE}$, the peak amplitude values are directly related to the damage modes. For instance, the most general trend is that the AE signals with an amplitude above $60-70 \mathrm{~dB}$ represent fiber breakage, 35-50 dB represents matrix cracking and 50-60 dB represents fiber debonding or delamination. There is no definite value for this correlation of the amplitude with damage modes. Different authors use different values based on their own experimental results. A summary of this information can be found in several literature $[3,20]$.

In recent years, however, some researchers have evidently argued that the AE signals with high amplitude not only represents the fiber breakage but also the interlaminar crack growth [4]. This is because of the mode of propagation and the degree of absorption of the AE waveforms. The peak amplitude only corresponds to the largest voltage peak and the modes of propagation and the degree 
of absorption in the propagation medium are ignored. For this reason, the I-Frequency and RFrequency are considered for this study.

Since the decaying frequency of the AE signals can be defined by the R-Frequency, while the IFrequency can define the characteristics of the initiation level before the largest amplitude is recorded. For this reason, in this research work, the peak amplitude is compared with the I-Frequency and R-Frequency for analysis.

\subsection{Fuzzy c-Means Data Clustering Results}

The I-Frequency and R-Frequency over the different amplitudes of the recorded AE events are clustered using the FCM clustering technique. Initially, for selecting the optimal number of clusters, the Davies-Bouldin Index (DBI) is calculated for the above-mentioned data. The DBI with the minimum value for the different clusters $N=1,2, \ldots, 6$ is supposed to be selected as the optimal number of clusters. For all sets of data recorded for SLS 1, SLS 2 and SLS 3, the DBI value returned the lowest for $N=3$. The FCM is used for clustering I-Frequency and R-Frequency into three clusters. The clustered data of I-Frequency and R-Frequency, respectively, over amplitude is presented in Figures 3 and 4 for all SLS specimens.

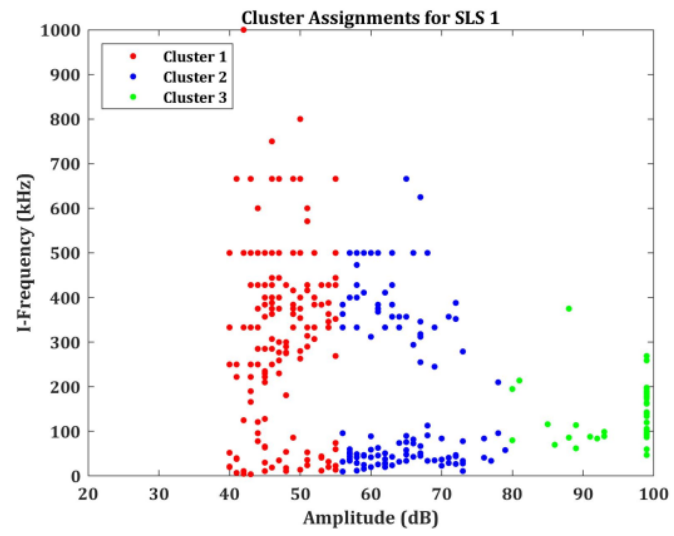

(a)

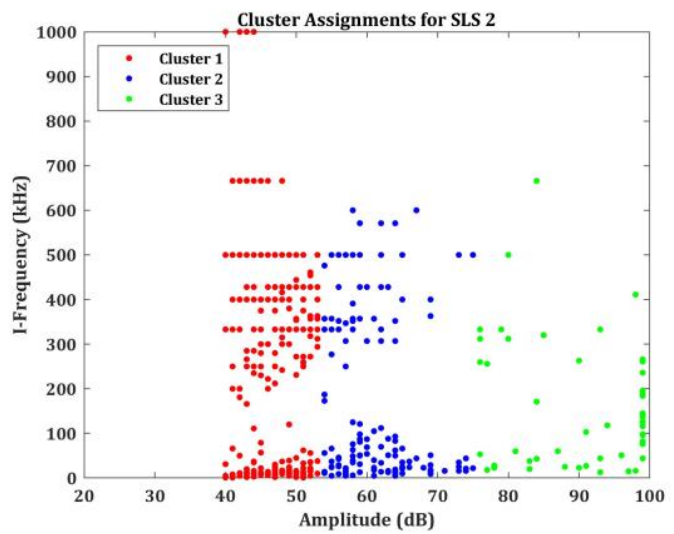

(b)

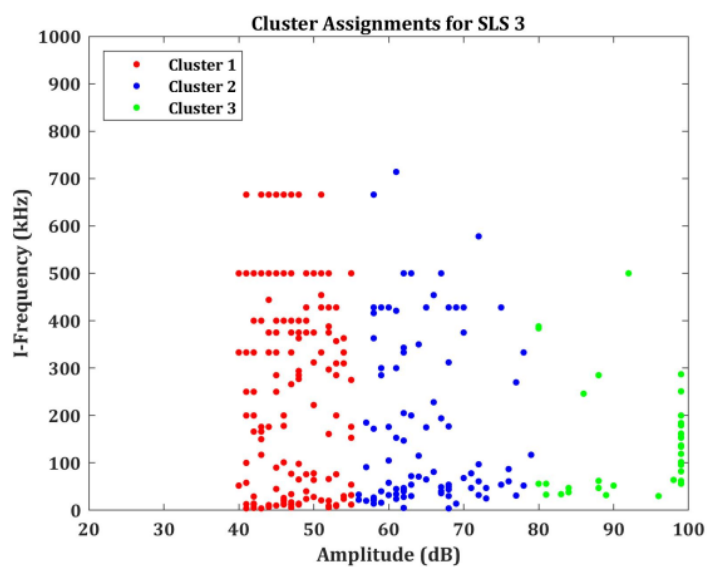

(c)

Figure 3. I-Frequency vs. Amplitude plotted clustered into 3 clusters using FCM for (a) SLS 1 (b) SLS 2 and (c) SLS 3 specimens.

The clustered data in Figure 3 shows that despite the peak amplitude level, the R-frequency of the recorded waveforms varies significantly. Similar results are also observed in Figure 4 for the RFrequency. Besides, the frequency levels in I-Frequency and R-Frequency in Figures 3 and 4 are not also very similar. This also means that the frequency rate of absorption, which can be indicated by the R-Frequency varies significantly from the I-Frequency. A question may arise, why the I- 
Frequency ranges from $0 \mathrm{kHz}$ to $1000 \mathrm{kHz}$, while the R-Frequency is only below $500 \mathrm{kHz}$. The IFrequency is calculated from Equation (4), which is the ratio of P.Counts and Risetime. If the second count or the third count of the AE waveform has the largest amplitude or magnitude in its FFT spectrum, then the P.Counts is counted as 1 or 2, respectively. This also means that there is a huge possibility that the Risetime can be very short during these instances. This is the reason for the wide range of I-Frequency compared to the R-Frequency.

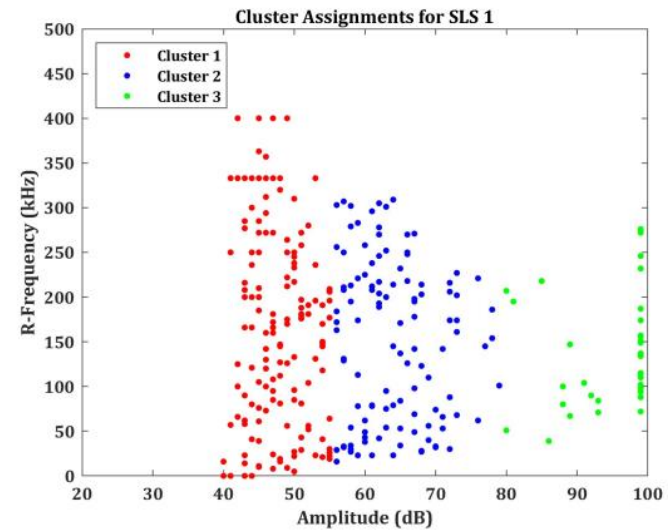

(a)

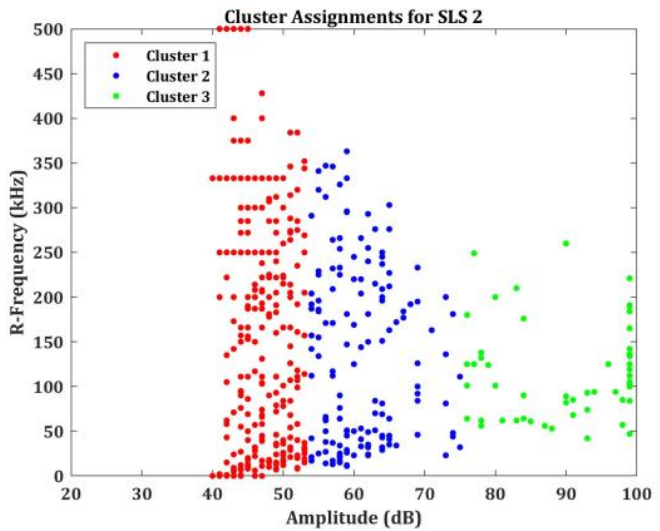

(b)

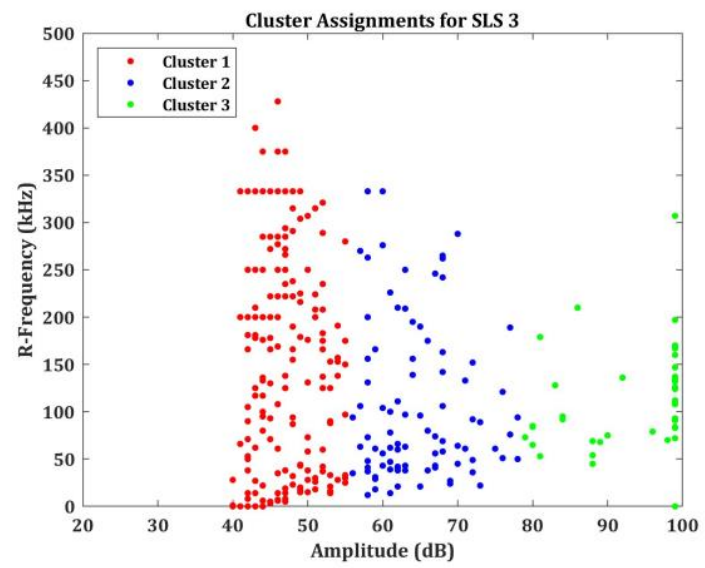

(c)

Figure 4. R-Frequency vs. Amplitude plotted clustered into 3 clusters using FCM for (a) SLS 1 (b) SLS 2 and (c) SLS 3 specimens.

The clusters in I-Frequency can be classified as follows: Cluster 1 has AE signals with low amplitude but with I-Frequency spread between $0 \mathrm{kHz}$ to $1000 \mathrm{kHz}$; Cluster 2 has AE signals with moderate amplitude (mostly between $55 \mathrm{~dB}$ and 75/80 dB) with I-Frequency spread between $0 \mathrm{kHz}$ and $700 \mathrm{kHz}$; Cluster 3 has the signals with higher amplitude $(>75 / 80 \mathrm{~dB})$ but with I-Frequency spread between $0 \mathrm{kHz}$ and $500 \mathrm{kHz}$, ignoring the outliers. Since I-Frequency is inversely proportional to the Risetime, it can be used as a parameter for classifying the type of damage mode, the AE signals have their source. Several reports have indicated the AE signals with larger peak amplitude and shorter Risetime correspond to the source as interlaminar crack, while the signals with smaller peak amplitude and longer Risetime may correspond to the shearing mode under the tensile loading. In analogous with that observation, the differences between the shearing mode and interlaminar crack can be identified $[3,5,7]$. A detailed explanation of how to identify the damage sources is presented in the next section.

The R-Frequency represents the amount of absorption of the AE signals. The AE signals generally propagate in two different modes: symmetrical and asymmetrical. The symmetrical mode AE signals carry a lower frequency component, but has less dispersion in its energy during the 
propagation. The low amount of dispersion means that the R-Frequency possibly can be high for symmetrical mode. The AE signals generated from matrix cracking and delamination release AE signals, which propagate in symmetric mode. On the contrary, the AE signals, which are propagating in asymmetric mode has a higher frequency and disperse more during propagation. The asymmetric mode AE signals possibly can have lower R-Frequency compared to the symmetric mode AE signals. These asymmetric AE signals mostly have fiber breakage or interlaminar crack growth as their $\mathrm{AE}$ source.

\subsection{Damage Assessment Using AE Descriptors}

For assessing the damage modes properly, it is essential to map the AE descriptors with the load response of the SLS specimens. The clustered AE data are plotted over the load response of the SLS specimens for detailed analysis. The SLS load response results with I-Frequency and amplitude are presented in Figure 5, and the results with R-Frequency and amplitude are presented in Figure 6.

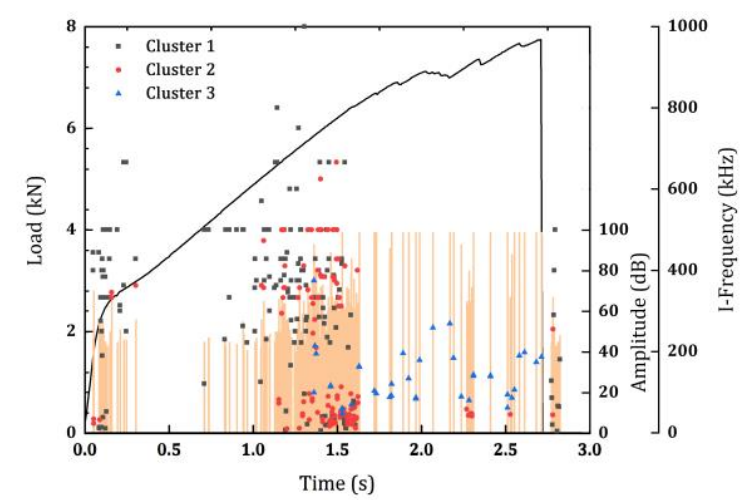

(a)

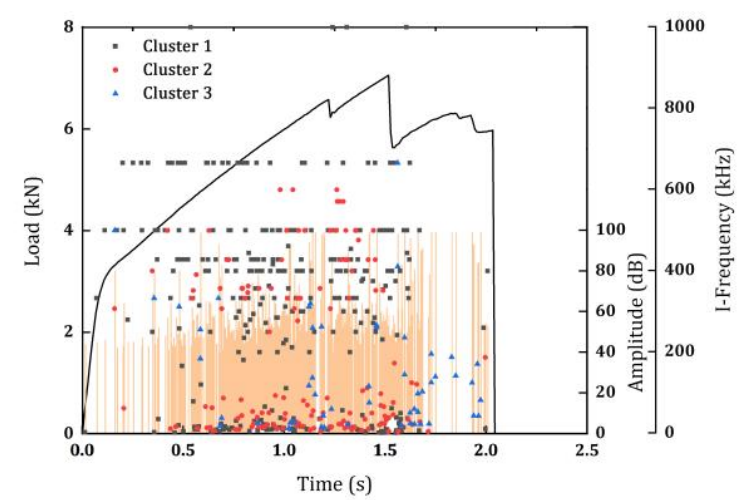

(b)

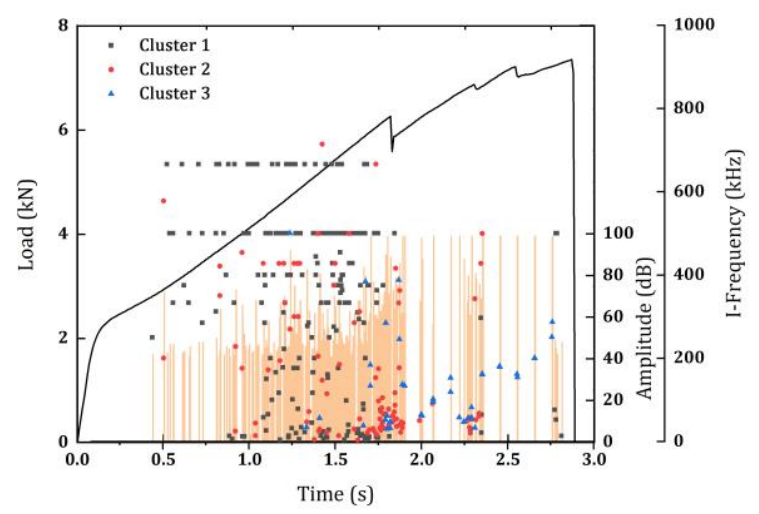

(c)

Figure 5. Load, Amplitude and Clustered I-Frequency vs Time for (a) SLS 1 (b) SLS 2 and (c) SLS 3 specimens.

In Figure 5a, the amplitude and clustered I-Frequency are plotted over the load response of the SLS 1 specimen. First of all, the load responses have multiples load peaks before the final fracture. In our previous works, the authors classified these peaks as regions of initial rupture and final rupture. For SLS 1, the initial rupture occurs at $7.05 \mathrm{kN}$ and the final rupture at $7.75 \mathrm{kN}$. From the point of initial rupture to the final rupture (roughly between $1.75 \mathrm{~s}$ and $2.75 \mathrm{~s}$, the majority of the I-Frequency components are in Cluster 3. Comparing this observation with Figure 3a, it can be identified that these clusters belong to the category of AE signals with high amplitude and low I-Frequency $(<200$ $\mathrm{kHz}$ ). Similarly, if the results are compared with Figure 6a and Figure 4a, again, the AE signals have 
low R-Frequency $(<200 \mathrm{kHz})$. This infers that the signals are highly symmetric since both I-Frequency and R-Frequency are below $200 \mathrm{kHz}$, at the same time, the peak amplitude is higher. These types of $\mathrm{AE}$ signals normally have interlaminar crack growth as their source. If the same Figures are compared for the initial stages of load response, say before $0.5 \mathrm{~s}$, the I-Frequency and R-Frequency are highly asymmetric. In our previous reports, the authors have indicated that this initial loading stage may represent the sliding of the specimen inside the loading grips [21]. The next important stage is the stage between the initial stage and the initial rupture (between $0.75 \mathrm{~s}$ and $1.75 \mathrm{~s}$ ). In Figure $5 \mathrm{a}$, most of the AE signals have I-Frequency in Cluster 1 and Cluster 2. A similar observation can be found in R-Frequency in Figure 6a. Owing to the absence of Cluster 3 in I-Frequency and R-Frequency, the damage modes in these regions are probably due to the matrix cracking and delamination. It has been indicated by several researchers that in adhesive bonded components, under static loading conditions, a majority of the load is carried by the adherends [22,23]. The delamination, however, will initiate at the vicinity of the adhesive layer and the adherend and extends through the thickness of the adhesive layer. The region before the initial rupture has suffered extensive microcracking in the thick adhesive layer and the delamination has initiated. However, the specimen SLS 1 retains its loadcarrying capability until it reaches $7.75 \mathrm{kN}$. During this transition stage, the crack had grown through the thickness, releasing AE signals with high symmetry between I-Frequency and R-Frequency, resulting in the final fracture.

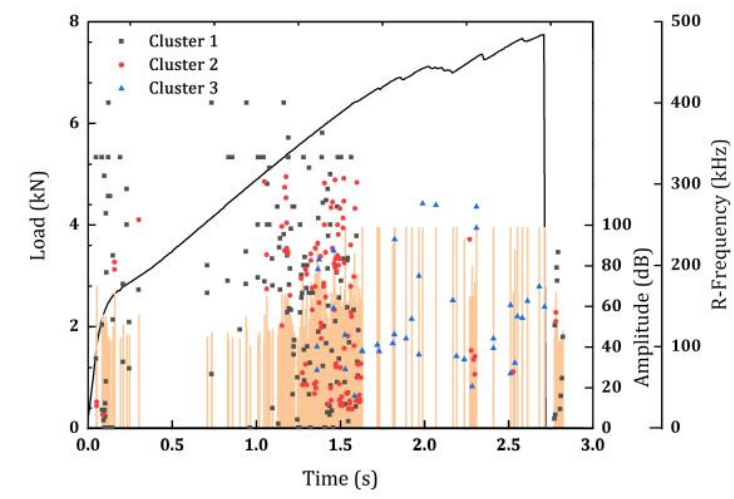

(a)

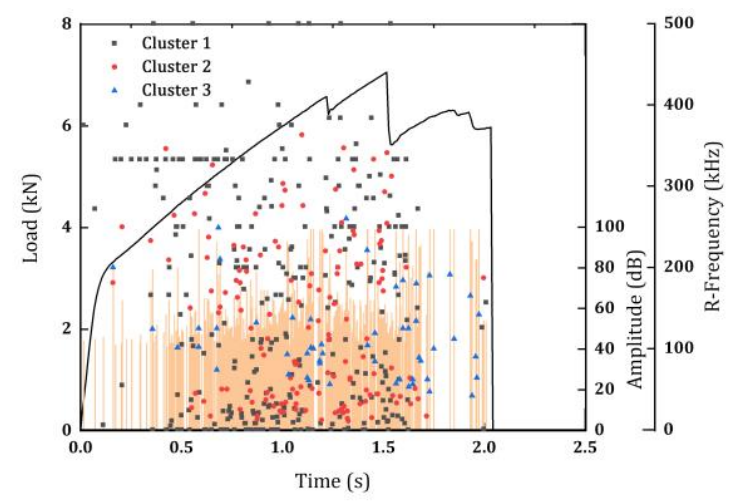

(b)

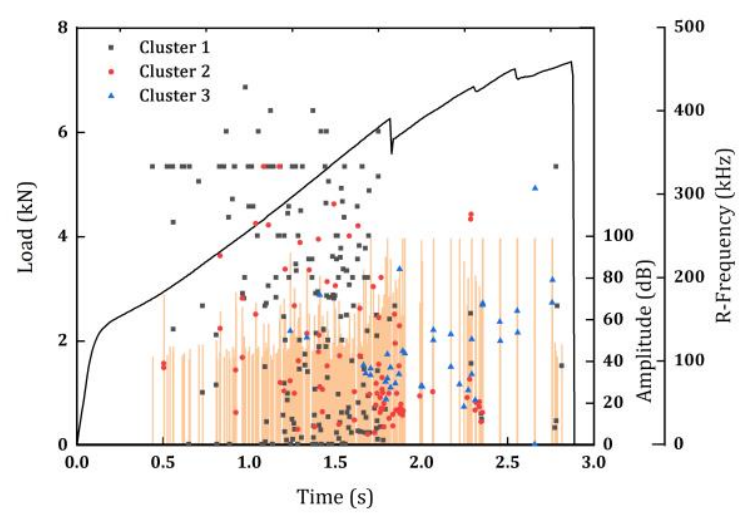

(c)

Figure 6. Load, Amplitude and Clustered R-Frequency vs Time for (a) SLS 1 (b) SLS 2 and (c) SLS 3 specimens.

In Specimen SLS 2 (Figures $5 \mathrm{~b}$ and $6 \mathrm{~b}$ ), more than one load peak can be observed. Nonetheless, the major initial rupture occurs at $1.5 \mathrm{~s}$ with the load peak of $7.05 \mathrm{kN}$ and the final rupture at $2.25 \mathrm{~s}$ at $5.96 \mathrm{kN}$. It is clear from this observation that the damage modes of SLS 2 are different from SLS 1. 
However, there is an ambiguity in how this damage mode has progressed. Comparing these results with the I-Frequency and R-Frequency clusters of Figures $3 \mathrm{~b}$ and $4 \mathrm{~b}$, the Cluster 3 signals of IFrequency and R-Frequency are distributed throughout the loading regions. Between the transition period of initial and final rupture, which extends only for $0.75 \mathrm{~s}$, a significant number of Cluster 3 signals are observed. Even though the stage is shorter in duration, these signals still refer to the interlaminar crack growth. The distribution of Cluster 3 throughout the loading stages indicates that the crack growth has initiated at a very early stage in SLS 2 . This is probably the reason for the very low final rupture load $(5.96 \mathrm{kN})$ compared to SLS 1 specimen.

The results of SLS 3 specimens are presented in Figures $5 c$ and $6 c$, which is almost identical to SLS 1. The interlaminar crack growth is observed between the initial and final rupture stages, which are at $5.93 \mathrm{kN}$ (at $1.75 \mathrm{~s}$ ) and $7.36 \mathrm{kN}$ (at $2.75 \mathrm{~s}$ ). The AE signals that correspond to the matrix cracking and delamination are distributed after the duration of $0.5 \mathrm{~s}$.

The I-Frequency and R-Frequency clustered data can provide information about the damage modes. More detailed analysis, such as counting the number of signals corresponds to matrix cracking and interlaminar crack growth and mapping them with the in-line fractographic analysis can provide detailed information about these parameters.

\section{Conclusions}

An Acoustic Emission multiparameter approach has been used for identifying the damage modes occurring in CFRP specimens configured in Single Lap Shear (SLS) configuration. First, from different $\mathrm{AE}$ features, the best features for analysis are selected by reducing their dimensions into principal components by PCA. Consequently, the Initiation Frequency (I-Frequency), Reverberation Frequency (R-Frequency) and Peak Amplitudes are chosen to be the best features for analysis. The selected features are clustered using the Fuzzy c-means (FCM) algorithm into three clusters. Each cluster has I-Frequency and R-Frequency distributed over their peak amplitudes. Then this clustered information is plotted over the load responses of the SLS specimens for identifying the damage modes. The future scope of this work is to extend this with an in-line fractographic analysis for directly relating the observed damage modes with the AE features. With this, the AE features can more effectively be used for damage analysis.

Author Contributions: conceptualization, P.K.V., C.B., and G.P.; methodology, P.K.V.; validation, P.K.V., C.B. and G.P.; formal analysis, P.K.V., C.B. and G.P.; investigation, P.K.V., C.B. and G.P.; data curation, P.K.V. and C.B.; writing-original draft preparation, P.K.V.; writing-review and editing, P.K.V., C.B. and G.P.; supervision, C.B. and C.C.; project administration, C.C. All authors have read and agreed to the published version of the manuscript.

Funding: This research received no external funding.

Conflicts of Interest: The authors declare no conflict of interest.

\section{References}

1. Giordano, M.; Condelli, L.; Nicolais, L. Acoustic emission wave propagation in a viscoelastic plate. Compos. Sci. Technol. 1999, 59, 1735-1743.

2. Sause, M.G.R. (Eds.) Acoustic Emission. In Situ Monitoring of Fiber-Reinforced Composites; Springer: Berlin/Heidelberg, Germany, 2016; Volume 242, p. 131.

3. Barile, C.; Casavola, C.; Pappalettera, G.; Vimalathithan, P.K. Application of different acoustic emission descriptors in damage assessment of fiber reinforced plastics: A comprehensive review. Eng. Fract. Mech. 2020, 235, 107083.

4. Oz, F.E.; Ersoy, N.; Lomov, S.V. Do high frequency acoustic emission events always represent fibre failure in CFRP laminates? Compos. Part A Appl. Manuf. 2017, 103, 230-235.

5. Barile, C.; Casavola, C.; Pappalettera, G.; Vimalathithan, P.K. Damage characterization in composite materials using acoustic emission signal-based and parameter-based data. Compos. Part B Eng. 2019, 178, 107469. 
6. Barile, C.; Casavola, C.; Pappalettera, G.; Vimalathithan, P.K. Damage assessment of carbon fibre reinforced plastic using acoustic emission technique: Experimental and numerical approach. Struct. Health Monit. 2020, doi:10.1177\%2F1475921720946438.

7. Aggelis, D.J.; Barkoula, N.M.; Matikas, T.E.; Paipetis, A.S. Acoustic structural health monitoring of composite materials: Damage identification and evaluation in cross ply laminates using acoustic emission and ultrasonics. Compos. Sci. Technol. 2012, 72, 1127-1133.

8. Kharrat, M.; Ramasso, E.; Placet, V.; Boubakar, M.L. A signal processing approach for enhanced Acoustic Emission data analysis in high activity systems: Application to organic matrix composites. Mech. Syst. Signal Process. 2016, 70-71, 1038-1055.

9. Guel, N.; Hamam, Z.; Godin, N.; Reynaud, P.; Caty, O.; Bouillon, F.; Paillassa, A. Data Merging of AE Sensors with Different Frequency Resolution for the Detection and Identification of Damage in OxideBased Ceramic Matrix Composites. Materials 2020, 13, 4691.

10. Soden, P.D.; Hinton, M.J.; Kaddour, A.S. Biaxial test results for strength and deformation of a range of Eglass and carbon fibre reinforced composite laminates: Failure exercise benchmark data. Compos. Sci. Technol. 2002, 62, 1489-1514.

11. Hinton, M.J.; Kaddour, A.S.; Soden, P.D. Evaluation of failure prediction in composite laminates: Background to "part B" of the exercise. Compos. Sci. Technol. 2002, 62, 1481-1488.

12. Hinton, M.J., Soden, P.D. Predicting failure in composite laminates: The background to the exercise. Compos. Sci. Technol. 1998, 58, 1001-1010.

13. Liptai, R.G.; Harris, D.O.; Tatro, C.A. (Eds.) An introduction to acoustic emission. In Acoustic Emission; ASTM STP 505; ASTM International: West Conshohocken, PA, USA, 1972; pp. 3-10.

14. Liptai, R.G. Acoustic emission from composite materials. In Composite Materials: Testing and Design; ASTM STP 497; Corten, H., Eds.; ASTM International: West Conshohocken, PA, USA, 1972; pp. 285-298.

15. Hamstad, M.A. Thirty years of advances and some remaining challenges in the application of acoustic emission to composite materials. In Acoustic Emission-Beyond the Millennium, Kishi, T., Ohtsu, M., Yuyama, S., Eds.; Elsevier: Oxford, UK, 2000; pp. 77-91.

16. ASTM D5868-01. Standard Test Method for Lap Shear Adhesion for Fiber Reinforced Plastic (FRP) 361 Bonding; ASTM International: West Conshohocken, PA, USA, 2014. Available online: www.astm.org (accessed on 6 November 2020).

17. Hotelling, H. Analysis of a complex of statistical variables into principal components. J. Educ. Psychol. 1933, 24, 417.

18. Maćkiewicz, A.; Ratajczak, W. Principal Component Analysis. Comput. Geosci. 1993, 19, 303-342.

19. Fotouhi, M.; Sadeghi, S.; Jalalvand, M.; Ahmadi, M. Analysis of the damage mechanisms in mixed-mode delamination of laminated composites using acoustic emission data clustering. J. Thermoplast. Compos. Mater. 2017, 30, 318-340.

20. Chandarana, N.; Sanchez, D.M.; Soutis, C.; Gresil, M. Early Damage Detection in Composites during Fabrication and Mechanical Testing. Materials 2017, 10, 685.

21. Barile, C.; Casavola, C.; Moramarco, V.; Pappalettere, C.; Vimalathithan, P.K. Bonding Characteristics of Single- and Joggled-Lap CFRP Specimens: Mechanical and Acoustic Investigations. Appl. Sci. 2020, $10,1782$.

22. Roderick, G.L.; Everett, R.A.; Crews, J.H. Debond propagation in composite-reinforced metals. In Fatigue of Composite Materials; ASTM International: West Conshohocken, PA, USA, 1975.

23. Mangalgiri, P.D.; Johnson, W.S.; Everett, R.A., Jr. Effect of adherend thickness and mixed mode loading on debond growth in adhesively bonded composite joints. J. Adhes. 1987, 23, 263-288.

Publisher's Note: MDPI stays neutral with regard to jurisdictional claims in published maps and institutional affiliations.

(C) 2020 by the authors. Submitted for possible open access publication under the terms and conditions of the Creative Commons Attribution (CC BY) license (http://creativecommons.org/licenses/by/4.0/). 